\title{
Continuously tuneable critical current in superconductor-ferromagnet multilayers
}

P. J. Curran, J. Kim, N. Satchell, J. D. S. Witt, G. Burnell, M. G. Flokstra, S. L. Lee, and S. J. Bending

Citation: Appl. Phys. Lett. 110, 262601 (2017); doi: 10.1063/1.4989693

View online: http://dx.doi.org/10.1063/1.4989693

View Table of Contents: http://aip.scitation.org/toc/apl/110/26

Published by the American Institute of Physics

\section{Articles you may be interested in}

Deterministic propagation of vortex-antivortex pairs in magnetic trilayers

Applied Physics Letters 110, 262402 (2017); 10.1063/1.4984898

Practical nanoscale field emission devices for integrated circuits Applied Physics Letters 110, 263101 (2017); 10.1063/1.4989677

Spin Hall magnetoresistance in an antiferromagnetic magnetoelectric $\mathrm{Cr}_{2} \mathrm{O}_{3}$ /heavy-metal W heterostructure Applied Physics Letters 110, 262401 (2017); 10.1063/1.4989680

Directly generating orbital angular momentum in second-harmonic waves with a spirally poled nonlinear photonic crystal

Applied Physics Letters 110, 261104 (2017); 10.1063/1.4990527

Subnanosecond microwave-assisted magnetization switching in a circularly polarized microwave magnetic field Applied Physics Letters 110, 262403 (2017); 10.1063/1.4986483

Electrode modulated capacitance-electric field nonlinearity in metal-insulator-metal capacitors Applied Physics Letters 110, 263503 (2017); 10.1063/1.4989531

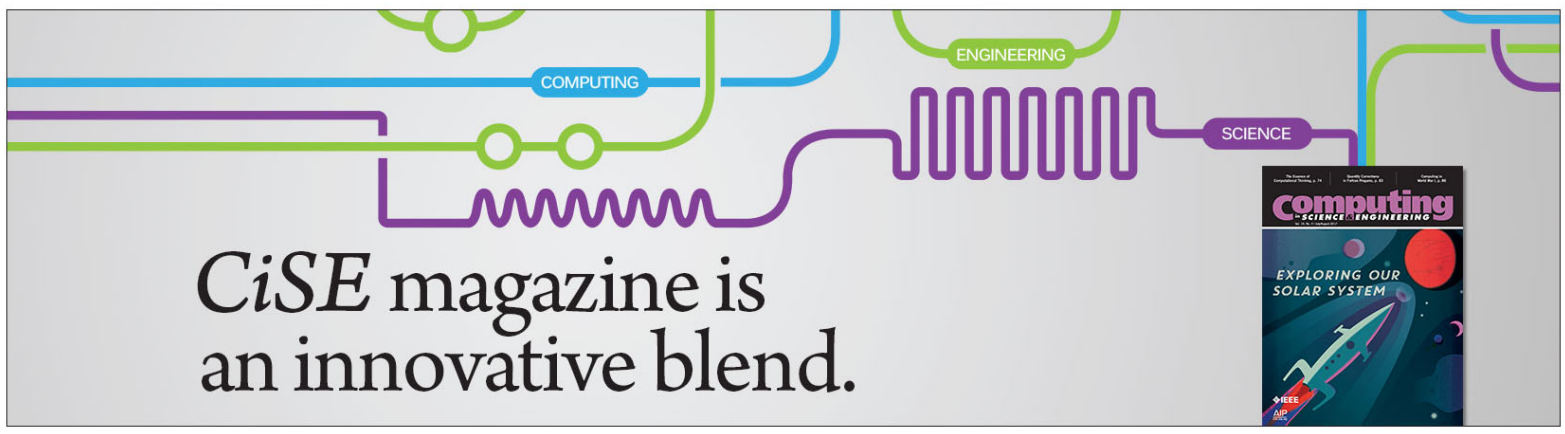




\title{
Continuously tuneable critical current in superconductor-ferromagnet multilayers
}

\author{
P. J. Curran, ${ }^{1}$ J. Kim, ${ }^{2}$ N. Satchell, ${ }^{2}$ J. D. S. Witt, ${ }^{2}$ G. Burnell, ${ }^{2}$ M. G. Flokstra, ${ }^{3}$ S. L. Lee ${ }^{3}$ \\ and S. J. Bending ${ }^{1}$ \\ ${ }^{1}$ Department of Physics, University of Bath, Claverton Down, Bath BA2 7AY, United Kingdom \\ ${ }^{2}$ School of Physics and Astronomy, University of Leeds, Leeds LS2 9JT, United Kingdom \\ ${ }^{3}$ School of Physics and Astronomy, SUPA, University of St. Andrews, St. Andrews KY16 9SS, United Kingdom
}

(Received 3 May 2017; accepted 11 June 2017; published online 27 June 2017)

\begin{abstract}
We demonstrate that the critical current of superconducting $\mathrm{Nb} / \mathrm{Ni}$ multilayers can be continuously tuned by up to a factor of three during magnetization reversal of the Ni films under an applied in-plane magnetic field. Our observations are in reasonably good agreement with a model of vortex pinning by Bloch domain walls that proliferate in the samples during magnetization reversal, whereby each vortex interacts with at most one wall in any of the Ni layers. Our model suggests ways in which the controllable pinning effect could be significantly enhanced, with important potential applications in tuneable superconducting devices. Published by AIP Publishing.

[http://dx.doi.org/10.1063/1.4989693]
\end{abstract}

Superconductivity and ferromagnetism have historically been viewed as antagonistic phenomena because their order parameters are normally incompatible. Over the last two decades, however, there has been a growing appreciation of the unique physical phenomena that can occur in hybrid superconductor-ferromagnet (S-F) structures. For heterostructures in direct electronic contact, this has led, for example, to the realization of $\pi$-junctions ${ }^{1}$ and long range spin-triplet supercurrents ${ }^{2}$ in superconductor-ferromagnetsuperconductor (SFS) structures. A different aspect of this problem is present in magnetically coupled heterostructures when vortex pinning in the superconductor is predicted to be greatly enhanced by the adjacent magnetic domain structure. This problem was addressed theoretically by Bulaevskii et $a l^{3}$ for an S-F multilayer when the ferromagnet has perpendicular magnetic anisotropy (PMA) and a stripe-like domain structure. In this case, domains of the appropriate sign of magnetization present a 1D pinning potential for vortices and are predicted to lead to a substantial increase in critical current. Several subsequent experiments on S-F bilayers have confirmed that the vortex pinning potential and critical current of the superconductor can be tuned by manipulating the domain structure of an adjacent ferromagnetic layer with PMA. ${ }^{4,5}$ Pinning of vortices by domain walls in S$\mathrm{F}$ bilayers, when the ferromagnetic layer has in-plane magnetic anisotropy, has also been considered. ${ }^{6}$ There have been fewer experimental studies of vortex pinning in S-F multilayers in this regime, although a number of groups have investigated the superconducting properties [e.g., $\mathrm{T}_{\mathrm{c}}, \mathrm{H}_{\mathrm{c} 2}(\mathrm{~T})$ ] of $\mathrm{Nb} / \mathrm{Ni}^{7}$ and $\mathrm{Nb} / \mathrm{Co}^{8}$ multilayers. In addition, Zhang et al. ${ }^{9}$ observed a significant enhancement of flux pinning in a $\mathrm{YBa}_{2} \mathrm{Cu}_{3} \mathrm{O}_{7}(30 \mathrm{~nm}) / \mathrm{Pr}_{0.67} \mathrm{Sr}_{0.33} \mathrm{MnO}_{3}$ (PSMO $30 \mathrm{~nm}$ ) bilayer of this type and proposed that vortices were pinned on the walls associated with the multidomain magnetic configuration in PSMO. Here, we demonstrate that the critical current (vortex pinning) in $\mathrm{Nb} / \mathrm{Ni}$ multilayers can be tuned by up to a factor of three upon applying an in-plane field to vary the magnetic domain structure of the Ni layers. Moreover, the superconducting parameters can be tuned continuously without reliance on specific (de-)magnetization cycles as is generally the case for $\mathrm{Co} / \mathrm{Pt}$ multilayers with PMA. ${ }^{5}$

In our multilayer samples, the magnetic structure of the Ni layers is almost independent of a small perpendicular component of magnetic field due their extremely high inplane magnetic anisotropy, while the rather long penetration depth in the disordered $\mathrm{Nb}$ thin films results in very weak screening of in-plane fields. Hence, we can effectively separate the roles of the in-plane and out-of-plane components of field; the former sets the ferromagnetic "template," and the latter induces superconducting vortices in the Nb layers. The ability to continuously tune critical currents in this way could find important applications in superconducting devices (e.g., memory cells) in which flux flow resistivity can be switched on and off.

$[\mathrm{Nb}(50 \mathrm{~nm}) / \mathrm{Ni}(\mathrm{x} \quad \mathrm{nm})]_{10}$ multilayers were grown on $\mathrm{Si}(100)$ substrates by magnetron sputtering in a deposition system with a base pressure of $\sim 10^{-8}$ mbar at a typical growth rate of $\sim 0.2 \mathrm{~nm} / \mathrm{s}$. X-ray reflectivity on otherwise identical multilayers indicates a Nb-Ni interface width of $<1.5 \mathrm{~nm}$, which is comparable to the surface roughness of sputter deposited single layer films of the same total thickness and indicates that these interfaces represent distinct structural boundaries. The time to move substrates between targets $(\sim 10 \mathrm{~s})$ is about a hundred times shorter than the estimated time for a monolayer of oxide to form at these pressures and the interfaces should be largely oxide free. Two different Ni layer thicknesses with different coercivities and magnetic roughness have been investigated $(x=5.5 \mathrm{~nm}$ and $\mathrm{x}=9.5 \mathrm{~nm})$ in order to study the influence that these have on the adjacent Niobium layers. The superconducting and ferromagnetic layers are in direct electronic contact in our samples, and the resulting exchangemediated pair breaking leads to a strong suppression of the critical temperature with respect to the $9.2 \mathrm{~K}$ value for bulk $\mathrm{Nb}$. Based on an extrapolation of the temperature-dependent irreversible magnetisation to zero, we estimate that $\mathrm{T}_{\mathrm{c}}=6.09 \pm 0.05 \mathrm{~K}$. 


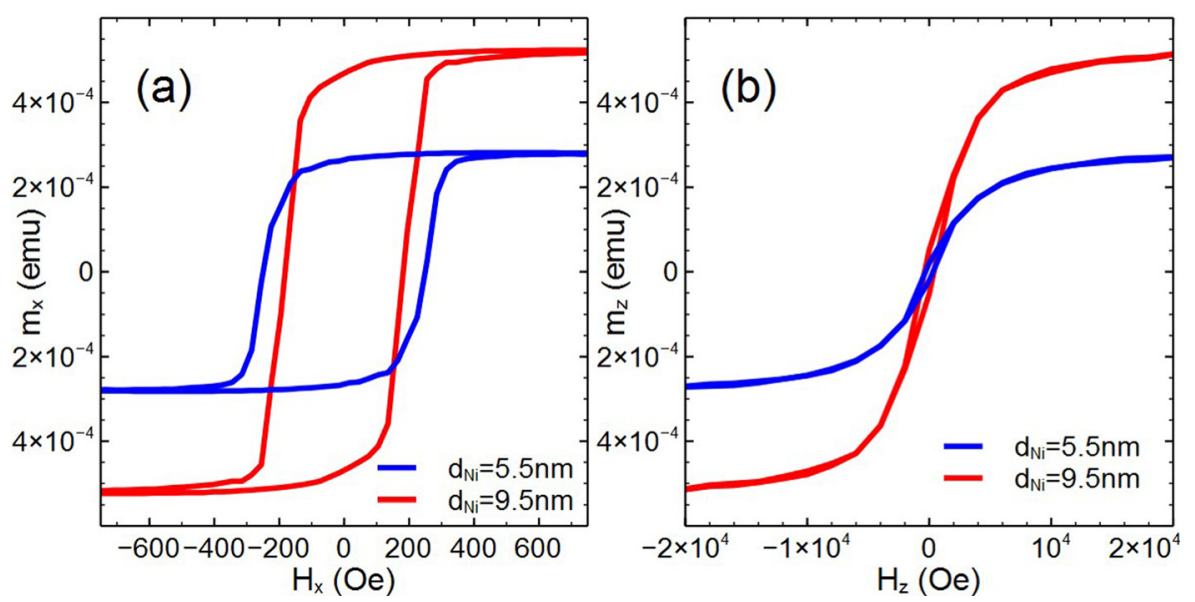

FIG. 1. SQUID magnetization of the two $\mathrm{Ni} / \mathrm{Nb}$ multilayer samples in different measurement geometries at $8 \mathrm{~K}$. (a) In-plane magnetization as a function of in-plane magnetic field. (b) Out-of-plane magnetization as a function of out-of-plane magnetic field.

High resolution scanning Hall microscopy $(\mathrm{SHM})^{10}$ has been used to directly image the stray fields at the surface of our multilayer samples at various temperatures down to $4.5 \mathrm{~K}$. Our microscope employs a custom-fabricated GaAs chip attached to the end of a piezoelectric scanner tube. The chip contains a nanoscale Hall sensor defined by the intersection of two $500 \mathrm{~nm}$ wide wires that have been lithographically patterned in the two-dimensional electron gas of a GaAs/AlGaAs heterostructure (Hall probe spatial resolution $\sim 500 \mathrm{~nm}$, minimum detectable field $\left.\sim 5 \mathrm{mG} /{ }_{\sqrt{ }} \mathrm{Hz}\right)$. This is situated $\sim 5 \mu \mathrm{m}$ from the Au-coated corner of a deep mesa etch that acts as an integrated scanning tunnelling microscope (STM) tip. The Hall probe is mounted at an angle of $\sim 1^{\circ}$ with respect to the sample plane to ensure that the STM tip is always the closest point to its surface. In operation the sample is approached towards the sensor until tunnelling is established and then retracted $\sim 100 \mathrm{~nm}$ for rapid "flying mode" scanning with the active Hall sensor $\sim 200-300 \mathrm{~nm}$ above the sample. In this way, quantitative maps of the component of magnetic induction perpendicular to the surface can be captured non-invasively. The Hall probe can also be parked just above the sample in a chosen location while an externally applied field is swept, allowing "local magnetization" loops to be captured.

Figures 1(a) and 1(b) show SQUID magnetization data for the two multilayer samples at $\mathrm{T}=8 \mathrm{~K}$ as a function of inplane $\left(\mathrm{H}_{\mathrm{x}}\right)$ and out-of-plane $\left(\mathrm{H}_{\mathrm{z}}\right)$ magnetic fields, respectively. The square hysteresis loops of Fig. 1(a) confirm the in-plane anisotropy of our samples, and Stoner-Wohlfarthtype fits ${ }^{11}$ to Fig. 1(b) incorporating second- and fourthorder uniaxial anisotropies yield the large anisotropy fields of $\mathrm{B}_{\mathrm{K}} \sim 1.1 \mathrm{~T}$ and $0.7 \mathrm{~T}$ for the $5.5 \mathrm{~nm}$ and $9.5 \mathrm{~nm}$ multilayer Ni samples, respectively.

Figure 2(a) shows local $\mathrm{B}_{\mathrm{z}}-\mathrm{H}_{\mathrm{x}}$ magnetization curves at $77 \mathrm{~K}$ generated by sweeping the in-plane field (parallel to the layers) with the Hall sensor parked just above the multilayer samples. Sharp peaks and dips in the range $\left|\mathrm{H}_{\mathrm{x}}\right| \sim 100-200$ Oe correspond to an uncorrelated (between Ni layers) network of domain walls sweeping under the Hall sensor as the magnetization reverses. Figures 2(b)-2(e) show selected sequential $77 \mathrm{~K}$ SHM images of the $\mathrm{x}(\mathrm{Ni})=9.5 \mathrm{~nm}$ multilayer taken at various points on the local magnetization loop of Fig. 2(a). Although the net Ni magnetization lies in the plane, we see quite strong perpendicular stray fields arising from regions where the magnetization tilts at a small angle out of the plane. This magnetic roughness is associated with the film granularity and appears to have a characteristic lengthscale of $\sim 1 \mu \mathrm{m}$. This is, however, close to the spatial resolution of our Hall sensor, and it is likely that the surface field has structure on
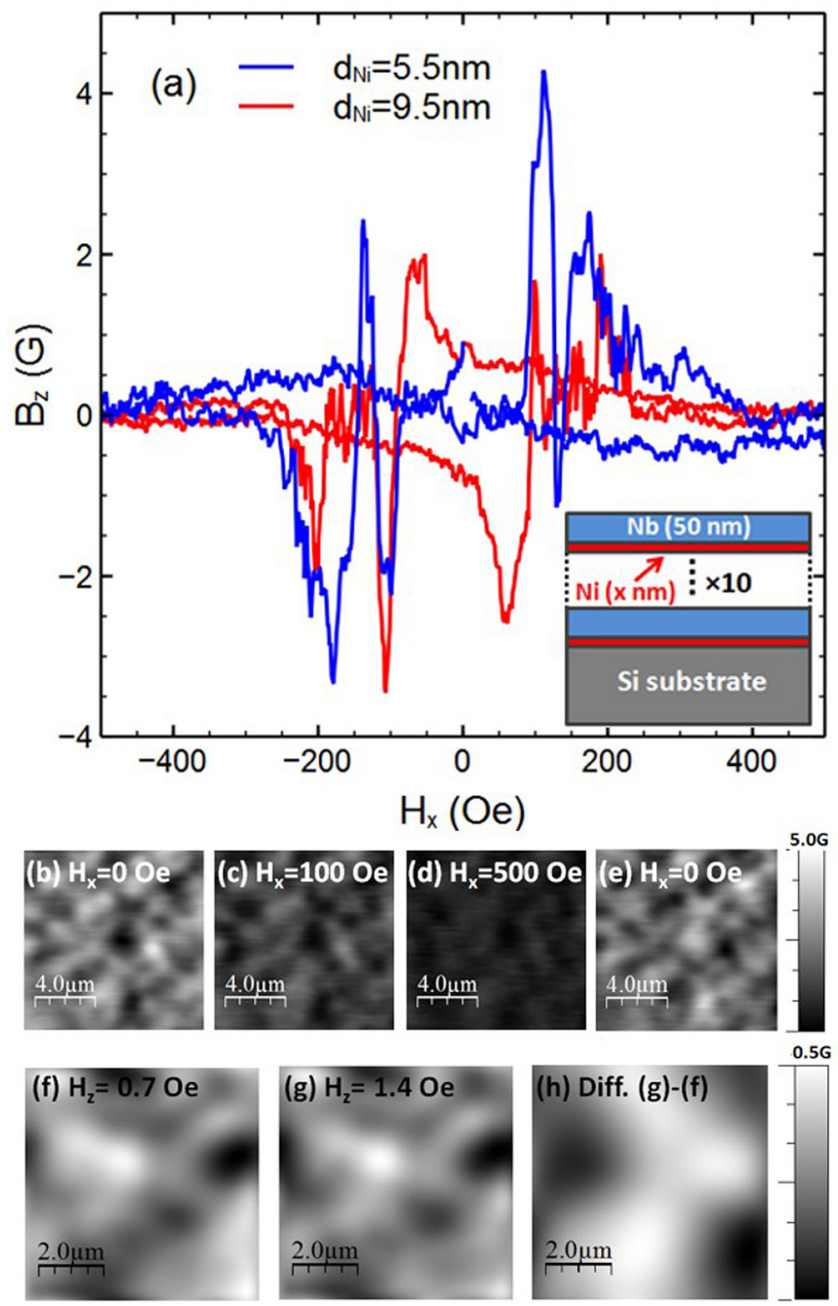

FIG. 2. (a) Local $\mathrm{B}_{\mathrm{Z}}-\mathrm{H}_{\mathrm{x}}$ magnetometry at $\mathrm{T}=77 \mathrm{~K}$ of the $\mathrm{d}_{\mathrm{Ni}}=5.5 \mathrm{~nm}$ and $\mathrm{d}_{\mathrm{Ni}}=9.5 \mathrm{~nm}$ multilayer samples. Inset shows schematic sample structure. (b)-(e) Sequence of SHM images at T $=77 \mathrm{~K}$ of the $\mathrm{d}_{\mathrm{Ni}}=9.5 \mathrm{~nm}$ multilayer at various applied in-plane fields through magnetic reversal. SHM images at $\mathrm{T}=4.5 \mathrm{~K}$ of the $\mathrm{d}_{\mathrm{Ni}}=5.5 \mathrm{~nm}$ sample at (f) $\mathrm{H}_{\mathrm{z}}=0.7 \mathrm{Oe}$ and $(\mathrm{g}) \mathrm{H}_{\mathrm{z}}=1.4 \mathrm{Oe}$. (h) Difference image obtained by numerically subtracting image (f) from image (g). 
much shorter lengthscales. In practice, domain wall motion during magnetization reversal is very rapid and extremely challenging to capture in a real space image. Here, we only resolve the magnetic roughness as a function of in-plane field, which clearly inverts contrast after magnetization reversal between $H_{x}=0$ [Fig. 2(b)] and $H_{x}=100$ Oe [Fig. 2(c)]. As the in-plane field is increased up to $500 \mathrm{Oe}$, we observe a steady decrease in the amplitude of the stray fields by a factor of almost three as the magnetization is trained back into the plane. The larger amplitude is recovered at remanence after the field has returned to zero [Fig. 2(e)].

Figures 2(f) and 2(g) show SHM images of the $\mathrm{x}(\mathrm{Ni})=5.5 \mathrm{~nm}$ sample after saturation with an in-plane field at $\mathrm{T}>\mathrm{T}_{\mathrm{c}}$, which was then removed before field-cooling to $4.5 \mathrm{~K}$ in $\mathrm{H}_{\mathrm{z}}=0.7 \mathrm{Oe}$ and $\mathrm{H}_{\mathrm{z}}=1.4 \mathrm{Oe}$, respectively. The $\mathrm{Nb}$ layers are superconducting at this temperature, and one would expect to be able to resolve different configurations of superconducting vortices threading the multilayer stacks. In practice, the images look superficially similar because the high level of residual magnetic roughness $(\sim 2 \mathrm{Oe})$ masks the weak vortex peak fields characteristic of the long penetration depth in our disordered $50 \mathrm{~nm} \mathrm{Nb}$ layers at this reduced temperature. However, when we numerically subtract the images to remove the magnetic roughness, which is unaffected by such small values of $\mathrm{H}_{\mathrm{z}}$, two or three broad white vortex structures with a peak amplitude of about $0.5 \mathrm{G}$ do become visible (Fig. 2(h)). This is close to the average number of vortices one would expect for these fields $\left(\mathrm{N} \sim \Delta \mathrm{B}_{\mathrm{z}} \mathrm{A} / \phi_{0}\right)$ and reveals a positional disorder due to pinning by the background magnetic roughness present in the nearby $\mathrm{Ni}$ layers.

Figure 3 displays typical local $\mathrm{B}_{\mathrm{z}}-\mathrm{H}_{\mathrm{z}}$ magnetization curves captured at fixed values of $\mathrm{H}_{\mathrm{x}}$ with the Hall sensor parked just above the surface of the $\mathrm{x}(\mathrm{Ni})=9.5 \mathrm{~nm}$ sample. We observe that the shape of these irreversible out-of-plane loops is a very strong function of the in-plane field applied, and this is the main result of our paper. These data have been

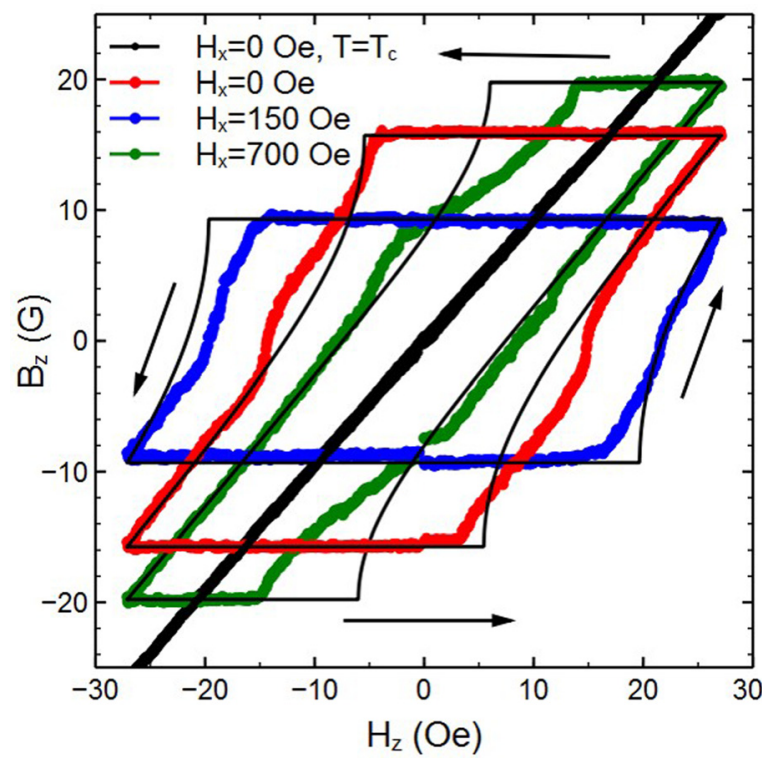

FIG. 3. Local $B_{z}-H_{z}$ magnetization loops for the $d_{N i}=9.5 \mathrm{~nm}$ multilayer at $\mathrm{T}=4.5 \mathrm{~K}$ and three different in-plane fields, $\mathrm{H}_{\mathrm{x}}$, and at $\mathrm{T}=\mathrm{T}_{\mathrm{c}}$ and $\mathrm{H}_{\mathrm{x}}=0$. Solid lines are fits to a model due to Brandt et al. (see text) and arrows indicate the direction of field sweep. analyzed using a model due to Brandt and Indenbom ${ }^{12}$ that was developed to describe the behavior of a thin type II superconducting strip of width $2 \mathrm{a}$ and thickness $\mathrm{d}$ in a perpendicular magnetic field, $\mathrm{H}_{\mathrm{a}}$. In the limit $\mathrm{d} \ll \mathrm{a}$, these authors showed that the zero field-cooled magnetic field profile $\mathrm{B}_{\mathrm{z}}(\mathrm{y})$ (where $\mathrm{y}$ is measured from the centre of the strip) at an applied field $\mathrm{H}_{\mathrm{a}}$ is described by

$$
\mathrm{B}_{\mathrm{z}}(\mathrm{y})= \begin{cases}0, & |\mathrm{y}|<\mathrm{b} \\ \mathrm{B}_{\mathrm{c}} \tan ^{-1} \frac{\sqrt{\left(\mathrm{y}^{2}-\mathrm{b}^{2}\right)}}{\mathrm{c}|\mathrm{y}|}, & \mathrm{b}<|\mathrm{y}|<\mathrm{a} .\end{cases}
$$

Here, $B_{c}=\mu_{0} j_{c} d / \pi$ ( $j_{c}$ is the critical current density of the Nb layers), $\mathrm{b}=\mathrm{a} / \cosh \left(\mu_{0} \mathrm{H}_{\mathrm{a}} / \mathrm{B}_{\mathrm{c}}\right)$ describes the outer perimeter of the flux-free region at the centre of the strip, and $\mathrm{c}=\tanh \left(\mu_{0} \mathrm{H}_{\mathrm{a}} / \mathrm{B}_{\mathrm{c}}\right)$. Furthermore, for an applied field cycle between the extremal values $\pm \mathrm{H}_{\mathrm{m}}$, it is shown that

$$
\mathrm{B}_{\mathrm{z}}^{\downarrow}\left(\mathrm{y}, \mathrm{H}_{\mathrm{a}}, \mathrm{j}_{\mathrm{c}}\right)=\mathrm{B}_{z}\left(\mathrm{y}, \mathrm{H}_{\mathrm{m}}, \mathrm{j}_{\mathrm{c}}\right)-\mathrm{B}_{z}\left(\mathrm{y}, \mathrm{H}_{\mathrm{m}}-\mathrm{H}_{\mathrm{a}}, 2 \mathrm{j}_{\mathrm{c}}\right) .
$$

The solid lines superimposed on the data in Fig. 3 are fits to Eqs. (1) and (2) with $\mathrm{a}=2.5 \mathrm{~mm}, \mathrm{~b}=0.5 \mathrm{~mm}, \mathrm{~d}=10 \times 50$ $=500 \mathrm{~nm}$, and $\mathrm{j}_{\mathrm{c}}$ as a free fit parameter. The analytical model describes our data rather well at high critical currents (cf. $\mathrm{H}_{\mathrm{x}}=0 \mathrm{Oe}$ and $\mathrm{H}_{\mathrm{x}}=150 \mathrm{Oe}$ ), though there are significant deviations at lower values of $\mathrm{j}_{\mathrm{c}}\left(\mathrm{cf} . \mathrm{H}_{\mathrm{x}}=700 \mathrm{Oe}\right.$ ) which may be related to the fact that the finite length $(\sim 8 \mathrm{~mm})$ of our samples breaks the model assumption of infinitely long strips.

Figure 4 plots the fitted values of $j_{c}$ for both the $\mathrm{x}(\mathrm{Ni})=5.5 \mathrm{~nm}$ and $\mathrm{x}(\mathrm{Ni})=9.5 \mathrm{~nm}$ multilayer samples as a function of fixed in-plane field. We find that the critical current shows a pronounced peak about the coercive field, driven by enhanced vortex pinning on the network of proliferating domain walls. Remarkably, we find that we can continuously tune $j_{c}$ with a constant in-plane field by up to a factor of three in this regime.

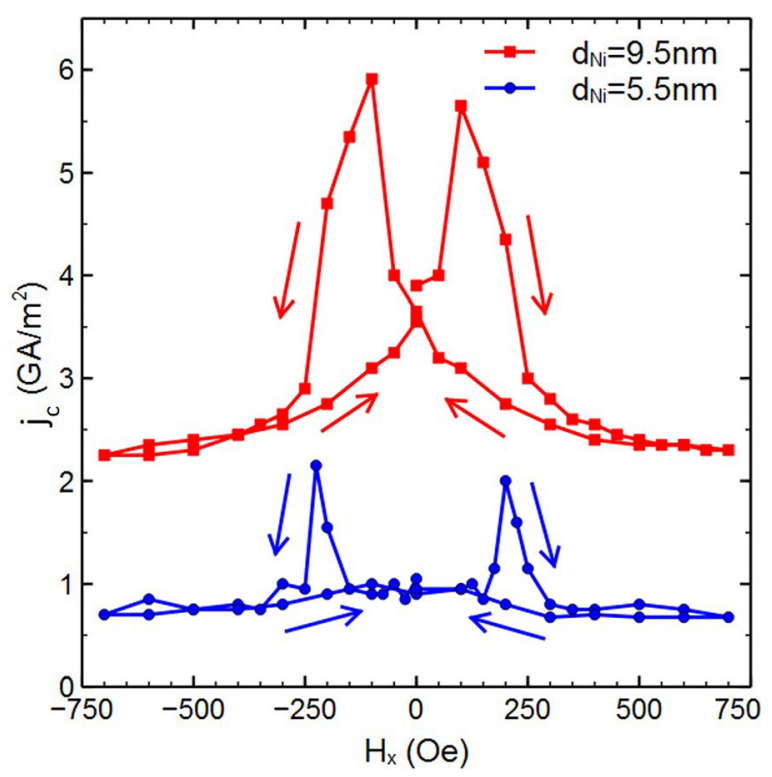

FIG. 4. Fitted values of $j_{c}$ as a function of in-plane magnetic field, $H_{x}$, for the two multilayer samples at $\mathrm{T}=4.5 \mathrm{~K}$. 
The $\mathrm{B}_{\mathrm{z}}-\mathrm{H}_{\mathrm{z}}$ irreversibility loops at constant $\mathrm{H}_{\mathrm{x}}$ plotted in Fig. 3, in practice, represent measurements under rotating magnetic fields, and it is not immediately clear that the model due to Brandt and Indenbom ${ }^{12}$ is a valid description of them. However, the ferromagnetic films in our $\mathrm{Nb} / \mathrm{Ni}$ multilayer samples have very high in-plane anisotropy [cf. Fig. 1(b)], and the magnetization state of the Ni layers is almost completely independent of the small perpendicular magnetic field component. In addition, the structural disorder in magnetron sputtered $\mathrm{Nb}$ films is known to lead to a thicknessdependent magnetic penetration depth. This was quantified by Gubin et al. ${ }^{13}$ who found $\lambda_{\mathrm{L}}(0) \sim 120 \mathrm{~nm}$ for similar $50 \mathrm{~nm}$ thick samples grown on $\mathrm{Si}$. Assuming a two-fluid model temperature-dependence we estimate a value of $\lambda_{\mathrm{L}}$ $=140 \mathrm{~nm}$ at the reduced temperature $\mathrm{T} / \mathrm{T}_{\mathrm{c}}=0.74$ of the measurements of Fig. 3, nearly three times larger than the individual $\mathrm{Nb}$ film thicknesses. Solutions of the London equation for magnetic fields parallel to the film predict very weak screening, and the field is only reduced by $\sim 1.6 \%$ in the centre of the $\mathrm{Nb}$ layers. Hence, the properties of the superconducting films are largely independent of the in-plane component of magnetic field, and the critical state model with a field perpendicular to the plane is indeed a reasonable approximation.

We turn now to the microscopic mechanism of the enhanced critical current observed. Although the out-ofplane magnetization associated with Bloch domain walls in our Ni films is much too small to nucleate vortex-antivortex pairs ${ }^{14}$ they can still act as strong vortex pinning sites. This is consistent with the observation that $\mathrm{j}_{\mathrm{c}}$ enhancements occur when the magnetization in the Ni films reverses and a network of "one-dimensional" magnetic domain walls is present. The interaction between a vortex line and a finite-sized magnetic particle is described by ${ }^{15}$

$$
\mathrm{U}_{\mathrm{p}}(\mathrm{r})=-\int_{\mathrm{V}_{\mathrm{f}}} \mathrm{M}\left(\mathrm{r}^{\prime}\right) \cdot \mathrm{B}_{\mathrm{v}}\left(\mathrm{r}-\mathrm{r}^{\prime}\right) \mathrm{d}^{3} \mathrm{r}^{\prime}
$$

where $M$ is the magnetization of the particle and $B_{v}$ is the magnetic induction at the vortex. For a vortex pinned on a Bloch wall of width $\Delta[\sim 50 \mathrm{~nm}$ (Ref. 16) $]$ in a Ni film of thickness $\mathrm{d}_{\mathrm{Ni}}$ the interaction is approximately given by $\mathrm{U}_{\mathrm{p}}$ $\sim \Delta \mathrm{d}_{\mathrm{Ni}} \lambda_{\mathrm{L}} \mathrm{M}_{\mathrm{sat}} \mathrm{B}_{\mathrm{v}}^{\text {peak }}$, where $\mathrm{M}_{\text {sat }}$ is the saturation magnetization of $\mathrm{Ni}$ and $\mathrm{B}_{\mathrm{v}}^{\text {peak }}$ is the peak vortex field. The vortex pinning force is related to the maximum gradient of $U_{p}$ and, for displacements perpendicular to the wall, is approximately given by

$$
\mathrm{f}_{\mathrm{p}} \sim \frac{\mathrm{U}_{\mathrm{p}}}{(\Delta / 2)}=2 \mathrm{~d}_{\mathrm{Ni}} \lambda_{\mathrm{L}} \mathrm{M}_{\mathrm{sat}} \mathrm{B}_{\mathrm{v}}^{\text {peak }} .
$$

$\mathrm{M}_{\text {sat }}=5 \times 10^{5} \mathrm{~A} / \mathrm{m}$ for $\mathrm{Ni}$, and using a thin film vortex model, ${ }^{17}$ we estimate that $\mathrm{B}_{\mathrm{v}}^{\text {peak }} \sim 20 \mathrm{mT}$ about $5 \mathrm{~nm}$ away from the $\mathrm{Nb}$ layer yielding $\mathrm{f}_{\mathrm{p}} \sim 27 \mathrm{pN}(15 \mathrm{pN})$ for a single adjacent $\mathrm{d}_{\mathrm{Ni}}=9.5 \mathrm{~nm}(5.5 \mathrm{~nm})$ layer. Using the standard expression to estimate the maximum experimental vortex pinning forces, $\mathrm{f}_{\mathrm{p}}=\mathrm{j}_{\mathrm{c}} \phi_{0} \mathrm{~d}_{\mathrm{Nb}} \sim 6 \mathrm{pN}$ and $\sim 2 \mathrm{pN}$ for the thicker and thinner samples, respectively. Given the approximations in our model, this level of agreement is reasonably good and the trend with Ni film thickness is well reproduced.
This suggests a picture whereby each vortex is pinned by at most one domain wall in one of the ten Ni layers. Bulaevskii et al. ${ }^{3}$ have considered the closely related problem of vortex pinning by an adjacent stripe ferromagnetic domain structure. This approximately maps onto our system if we replace their domain width by the domain wall width, and their domain period by the mean spacing between domain walls in any of the Ni layers. Accounting for the interplay between vortex pinning and vortex-vortex interactions, these authors derive a criterion for strong magnetic pinning, $2 \pi \sqrt{\mathrm{C}_{11} \mathrm{C}_{66}} /$ $\left(\mathrm{q}^{2} \mathrm{U}_{\mathrm{p}}\right) \leq 1$, with the pinning strength dropping rapidly when the inequality is no longer satisfied. Here, $C_{11}=B^{2} / 4 \pi$ is the vortex lattice compression modulus, $\mathrm{C}_{66}=\mathrm{B} \phi_{0} /\left(8 \pi \lambda_{\mathrm{L}}\right)^{2}$ is the shear modulus, and $q=2 \pi / 1$ is the wave vector of the domain wall potential (assumed 1D periodic with spacing 1). Applying this criterion to our system, we find that the mean lateral spacing between any pair of domain walls at magnetization reversal cannot be very much larger than 3-6 $\mu \mathrm{m}$.

In conclusion, we have demonstrated that the critical current of $\mathrm{Nb} / \mathrm{Ni}$ multilayers can be tuned by up to a factor of three during magnetization reversal of the Ni films under an in-plane magnetic field. Our observations are in reasonably good agreement with a model of vortex pinning by Bloch domain walls, whereby each vortex interacts with at most one wall in any of the Ni layers. The relatively strong average pinning potential observed allows us to estimate that the mean lateral spacing between domain walls at the peak critical current is no larger than 3-6 $\mu \mathrm{m}$ (about 3-6 mean vortex spacings at the maximum applied field of $2.7 \mathrm{mT}$ ). Vortex pinning on domain walls will also occur in samples with less repeat layers, even bilayers, but the influence on the critical current is expected to be very much weaker. Conversely, it should be possible to significantly enhance the effect by increasing the number of repeat units and/or the thickness of the Ni films in the multilayer. Importantly $\mathrm{j}_{\mathrm{c}}$ can be tuned continuously in our system and we do not rely on specific demagnetization cycles to get different domain configurations. Such structures could find applications in superconducting devices (e.g., memory cells) whereby one can switch from a dissipative to a non-dissipative state by the application of an in-plane magnetic field, or single photon detectors with tuneable critical currents.

The authors acknowledge financial support from EPSRC, UK, under Grant Nos. EP/G036101/1, EP/J010626/ 1, EP/J010634/1, EP/J01060X/1, and EP/I031014/1; the NanoSC COST Action MP-1201; a JEOL Europe studentship under Grant No. EP/I000933/1, plus support from the STFC ISIS neutron and muon source.

All data discussed in this manuscript can be found at https://doi.org/10.15125/BATH-00386.

\footnotetext{
${ }^{1}$ V. V. Ryazanov, V. A. Oboznov, A. Yu. Rusanov, A. V. Veretennikov, A. A. Golubov, and J. Aarts, Phys. Rev. Lett. 86, 2427 (2001).

${ }^{2}$ R. S. Keizer, S. T. B. Goennenwein, T. M. Klapwijk, G. Miao, G. Xiao, and A. Gupta, Nature 439, 825 (2006); T. S. Khaire, M. A. Khasawneh, W. P. Pratt, Jr., and N. O. Birge, Phys. Rev. Lett. 104, 137002 (2010); J. W. Robinson, J. D. Witt, and M. G. Blamire, Science 329, 5987 (2010).

${ }^{3}$ L. N. Bulaevskii, E. M. Chudnovsky, and M. P. Maley, Appl. Phys. Lett. 76, 2594 (2000).

${ }^{4}$ A. García-Santiago, F. Sánchez, M. Varela, and J. Tejada, Appl. Phys.
} Lett. 77, 2900 (2000). 
${ }^{5}$ M. Lange, M. J. Van Bael, V. V. Moshchalkov, and Y. Bruynseraede, Appl. Phys. Lett. 81, 322 (2002); V. K. Vlasko-Vlasov, U. Welp, A. Imre, D. Rosenmann, J. Pearson, and W. K. Kwok, Phys. Rev. B 78, 214511 (2008); L. Y. Zhu, M. Z. Cieplak, and C. L. Chien, ibid. 82, 060503(R) (2010); M. Z. Cieplak, L. Y. Zhu, Z. Adamus, M. Kończykowski, and C. L. Chien, ibid. 84, 020514(R) (2011).

${ }^{6}$ I. S. Burmistrov and N. M. Chtchelkatchev, Phys. Rev. B 72, 144520 (2005).

${ }^{7}$ J. E. Mattson, R. M. Osgood, C. D. Potter, C. H. Sowers, and S. D. Bader, J. Vac. Sci. Technol. A 15, 1774 (1997); J. E. Villegas, E. Navari, D. Jaque, E. M. González, J. I. Martín, and J. L. Vicent, Physica C 369, 213 (2002); S. A. Kryukov, L. E. De Long, E. Navarro, J. E. Villegas, E. M. Gonzalez, and J. L. Vicent, IEEE Trans. Magn. 39, 2693 (2003); V. Shelukhin, A. Tsukernik, M. Karpovski, Y. Blum, K. B. Efetov, A. F. Volkov, T. Champel, M. Eschrig, T. Löfwander, G. Schön, and A. Palevski, Phys. Rev. B 73, 174506 (2006).
${ }^{8}$ C. Monton, F. de la Cruz, and J. Guimpel, Phys. Rev. B 75, 064508 (2007); 77, 104521 (2008).

${ }^{9}$ X. X. Zhang, G. H. Wen, R. K. Zheng, G. C. Xiong, and G. J. Lian, Europhys. Lett. 56, 119 (2001).

${ }^{10}$ A. Oral, S. J. Bending, and M. Henini, App. Phys. Lett. 69, 1324 (1996).

${ }^{11}$ W. Sucksmith and J. E. Thompson, Proc. R. Soc. London Ser. A 225, 362 (1954).

${ }^{12}$ E. H. Brandt and M. Indenbom, Phys. Rev. B 48, 12893 (1993).

${ }^{13}$ A. I. Gubin, K. S. Il'in, S. A. Vitusevich, M. Siegel, and N. Klein, Phys. Rev. B 72, 064503 (2005).

${ }^{14}$ M. V. Milosević and F. M. Peeters, Phys. Rev. B 68, 024509 (2003).

${ }^{15}$ A. Y. Aladyshkin, A. V. Silhanek, W. Gillijns, and V. V. Moshchalkov, Supercond. Sci. Technol. 22, 053001 (2009).

${ }^{16}$ C. T. Hsieh, J. Q. Liu, and J. T. Lue, Appl. Surf. Sci. 252, 1899 (2005).

${ }^{17}$ A. M. Chang, H. D. Hallen, H. F. Hess, H. L. Koa, J. Kwo, A. Sudbo, and T. Y. Chang, Europhys. Lett. 20, 645 (1992). 